\title{
REFERENCES
}

1. H. Busemann, Geometries in which the planes minimize area, Ann. Mat. Pura Appl. 55 (1961), 171-189.

2. - The geometry of geodesics, Academic Press, New York, 1955.

3. J. Szenthe, Über metrische Räume, deren lokalisometrische Abbildungen Isometrien sind, Acta Math. Acad. Sci. Hungar. 13 (1962), 433-441.

University of California, Riverside

\section{$A$-GENUS AND INDECOMPOSABILITY OF DIFFERENTIABLE MANIFOLDS}

\section{YASURÔ TOMONAGA}

Introduction. In the previous paper [1] we have studied the conditions on which a differentiable manifold be indecomposable and cited many examples of indecomposable manifolds. In this paper we shall study the relations between $A$-genus and indecomposability of a differentiable manifold.

1. Hereafter we denote by $X_{n}$ an $n$-dimensional compact orientable differentiable manifold. If $X_{n}=X_{r} \cdot X_{8}$ we say that $X_{n}$ is decomposable and if not, we say that $X_{n}$ is indecomposable. If $X_{4 n}=X_{n} \cdot X_{8}$ we have

$$
A\left(X_{4 n}\right)=A\left(X_{r}\right) A\left(X_{s}\right) ;
$$

where $A(X)$ denotes the $A$-genus of $X$ and we define as follows:

$$
A\left(X_{n}\right)=0, \quad n \not \equiv 0 \bmod 4 .
$$

If $r$ and $s$ are divisible by 4, the relation (1.1) follows from the general property of multiplicative series $[2$, p. 75]. According to the cobordism theory, the cobordism components of $X_{r}(r \neq 0$ mod 4$)$ consist only of torsions. Hence the product $X_{r} \cdot X_{s}$ also consists only of torsions. Therefore $A\left(X_{r} \cdot X_{s}\right)$ is zero. Thus (1.1) holds in general. Meanwhile Atiyah and Hirzebruch have proved the following:

Theorem 1 (Atiyah and Hirzebruch [3]). If $X_{4 n}$ is differentiably imbedded in the $(8 n-2 q)$-sphere, then $A\left(X_{4 n}\right)$ is divisible by $2^{q+1}$. If moreover $q \equiv 2 \bmod 4$, then $A\left(X_{4 n}\right)$ is divisible by $2^{q+2}$.

It is well known that an $X_{n}$ is always differentiably imbedded in the $2 n$-sphere. Hence we have from the above theorem

Received by the editors March 20,1963. 


$$
A\left(X_{4 n}\right) \equiv 0 \bmod 2 .
$$

We have from (1.1) and (1.3):

Theorem 2. If $A\left(X_{4 n}\right) \equiv 2 \bmod 4$, such an $X_{4 n}$ is indecomposable.

For example $P_{2^{s}}(c)$ is indecomposable, because

$$
A\left(P_{2 m}(c)\right)=(-1)^{m}\left(\begin{array}{c}
2 m \\
m
\end{array}\right)
$$

(see [3]). We have proved the above theorem for the cases where $n=2,3,4$. We shall find another example of Theorem 2 in the next paragraph.

2. Let $P_{2 m+1}(c)$ be the complex projective space of complex dimension $2 m+1$. The total Pontryagin class of $P_{2 m+1}(c)$ takes the form

$$
p=\left(1+g^{2}\right)^{2 m+2}, \quad g \in H^{2}\left(p_{2 m+1}(c), Z\right) .
$$

Let $X_{4 m}$ be a compact orientable differentiable submanifold of $P_{2 m+1}(c)$ and let $\lambda g$ be the cohomology class corresponding to the homology class represented by $X_{4 m}$. The $A$-genus of $X_{4 m}$ is given by

$$
A\left(X_{4 m}\right)=\prod_{i} \frac{2 \sqrt{ } \nu_{i}}{\sinh 2 \sqrt{ } \nu_{i}}
$$

where

$$
p\left(X_{4 m}\right)=\prod_{i}\left(1+\nu_{i}\right)
$$

Hence we have

$$
A\left(X_{4 m}\right)=\left[\frac{\sinh 2 \lambda g}{2}\left(\frac{2 g}{\sinh 2 g}\right)^{2 m+2}\right]\left[P_{2 m+1}(c)\right] \quad[2, \text { p. 86] }
$$

\section{Taking}

$$
g^{2 m+1}\left[P_{2 m+1}(c)\right]=1
$$

into account, we have

$$
A\left(X_{4 m}\right)=\frac{1}{2 \pi i} \int \frac{1}{z^{2 m+2}}\left(\frac{\sinh 2 \lambda z}{2}\right)\left(\frac{2 z}{\sinh 2 z}\right)^{2 m+2} d z
$$

where the integrand should be integrated along a small circle around $z=0$ anti-clockwise. Changing the variable as follows:

$$
\sinh 2 z=t
$$


we have

$$
\begin{aligned}
A\left(X_{4 m}\right) & =\frac{2^{2 m}}{2 \pi i} \int \frac{\sinh 2 \lambda z}{t^{2 m+2}\left(1+t^{2}\right)^{1 / 2}} d t \\
& =\frac{2^{2 m}}{2 \pi i} \int \frac{\left(\left(1+t^{2}\right)^{1 / 2}+t\right)^{\lambda}-\left(\left(1+t^{2}\right)^{1 / 2}-t\right)^{\lambda}}{2 t^{2 m+2}\left(1+t^{2}\right)^{1 / 2}} d t
\end{aligned}
$$

where the integrand should be integrated along the small circle around $t=0$ anti-clockwise. We have from (2.8):

$$
\begin{aligned}
A\left(X_{4 m}\right)= & \frac{2^{2 m}}{2 \pi i} \int \frac{1}{t^{2 m+2}}\left\{\lambda t\left(1+t^{2}\right)^{(\lambda / 2)-1}+\left(\begin{array}{c}
\lambda \\
3
\end{array}\right) t^{8}\left(1+t^{2}\right)^{(\lambda / 2)-2}\right. \\
& \left.+\left(\begin{array}{c}
\lambda \\
5
\end{array}\right) t^{5}\left(1+t^{2}\right)^{(\lambda / 2)-3}+\cdots\right\} d t \\
= & 2^{2 m}\left\{\lambda\left(\begin{array}{c}
(\lambda / 2)-1 \\
m
\end{array}\right)+\left(\begin{array}{c}
\lambda \\
3
\end{array}\right)\left(\begin{array}{c}
(\lambda / 2)-2 \\
m-1
\end{array}\right)\right. \\
& \left.\quad+\left(\begin{array}{c}
\lambda \\
5
\end{array}\right)\left(\begin{array}{c}
(\lambda / 2)-3 \\
m-2
\end{array}\right)+\cdots+\left(\begin{array}{c}
\lambda \\
2 m+1
\end{array}\right)\right\} \\
= & 2^{2 m} \lambda(\lambda-2) \cdots(\lambda-2 m)\left\{\frac{1}{2^{m} m !}+\frac{\lambda-1}{3 ! 2^{m-1}(m-1) !}\right. \\
& \left.\quad+\cdots+\frac{(\lambda-1)(\lambda-3) \cdots(\lambda-2 m+1)}{(2 m+1) !}\right\} \\
= & 2^{2 m} \lambda f(\lambda) .
\end{aligned}
$$

It is clear from (2.8) and (2.9) that

$$
f(\lambda)=f(-\lambda)
$$

and

$$
\begin{aligned}
f(2) & =f(4)=\cdots=f(2 m)=f(-2) \\
& =f(-4)=\cdots=f(-2 m)=0 .
\end{aligned}
$$

Therefore $f(\lambda)$ takes the form

(2.12) $f(\lambda)=c(\lambda-2)(\lambda-4) \cdots(\lambda-2 m)(\lambda+2)(\lambda+4) \cdots(\lambda+2 m)$.

Letting $\lambda=1$ in (2.9) we have

$$
c=1 / 2^{m} m ! 3 \cdot 5 \cdots(2 m+1) .
$$

Hence we have from (2.9), (2.12) and (2.13) 


$$
A\left(X_{4 m}\right)=\frac{2^{m} \lambda}{m !} \prod_{i=1}^{m}\left(\frac{\lambda^{2}-4 i^{2}}{2 i+1}\right) .
$$

If $m=2^{s}(s \geqq 1)$ and $\lambda$ is odd we have from (2.14)

$$
A\left(X_{4 m}\right) \equiv 2 \bmod 4 .
$$

Hence we have from Theorem 2

THEOREM 3. Let $X_{4 m}$ be a submanifold of $P_{2 m+1}(c)$ corresponding to $\lambda g$. Then $X_{4 m}$ is indecomposable provided that $m=2^{s}(s \geqq 1)$ and $\lambda$ is odd.

Next we consider the case where $w_{2}=0$. It is known that if $w_{2}=0$, then $\hat{A}\left(X_{4 n}\right)=2^{4 n} A\left(X_{4 n}\right)$ is an integer, i.e., $A\left(X_{4 n}\right)$ is divisible by $2^{4 n}[4]$. We see from (2.14) that if $w_{2}=0$, then $\lambda$ must be even. Thus we have

Theorem 4. If a submanifold $X_{4 n}$ of $P_{2 m+1}(c)$ corresponding to $\lambda g$ has the vanishing $w_{2}$, then $\lambda$ is even.

\section{REFERENCES}

1. Y. Tomonaga, Indecomposability of differentiable manifolds, Tôhoku Math. J. 14 (1962), 328-331.

2. F. Hirzebruch, Neue topologische Methoden in der algebraischen Geometrie, Springer, Berlin, 1956.

3. M. F. Atiyah et F. Hirzebruch, Quelques théorèmes de non-plongement pour les variêtés différentiables, Bull. Soc. Math. France 87 (1959), 383-396.

4. A. Borel and F. Hirzebruch, Characteristic classes and homogeneous spaces, Vol. III, Amer. J. Math. 82 (1960), 491-504.

UtSUNOMIYA UNIVERSITY, UTSUNOMIYA, JAPAN 